\title{
Erratum to: The role of stereotactic radiosurgery in the management of patients with newly diagnosed brain metastases: a systematic review and evidence-based clinical practice guideline
}

\author{
Mark E. Linskey - David W. Andrews • Anthony L. Asher • Stuart H. Burri • \\ Douglas Kondziolka - Paula D. Robinson - Mario Ammirati - Charles S. Cobbs • \\ Laurie E. Gaspar · Jay S. Loeffler • Michael McDermott • Minesh P. Mehta • \\ Tom Mikkelsen · Jeffrey J. Olson • Nina A. Paleologos • Roy A. Patchell • \\ Timothy C. Ryken $\cdot$ Steven N. Kalkanis
}

Published online: 1 January 2010

(C) Springer Science+Business Media, LLC. 2009

Erratum to: J Neurooncol

DOI 10.1007/s11060-009-0073-4

In the Absract of the original publication, the level numbers indicated for the Recommendations regarding $\boldsymbol{S R S}$ plus WBRT vs. WBRT alone were incorrect. The correct level numbers are shown below.

\section{Recommendations}

SRS plus WBRT vs. WBRT alone

Level 1 Single-dose SRS along with WBRT leads to significantly longer patient survival compared with WBRT

The online version of the original article can be found under doi:10.1007/s11060-009-0073-4.

\section{E. Linskey}

Department of Neurosurgery, University of California-Irvine

Medical Center, Orange, CA, USA

D. W. Andrews

Department of Neurosurgery, Thomas Jefferson University,

Philadelphia, PA, USA

\author{
A. L. Asher \\ Department of Neurosurgery, Carolina Neurosurgery and Spine \\ Associates, Charlotte, NC, USA \\ S. H. Burri \\ Department of Radiation Oncology, Carolinas Medical Center, \\ Charlotte, NC, USA \\ D. Kondziolka \\ Department of Neurological Surgery, University of Pittsburgh \\ Medical Center, Pittsburgh, PA, USA \\ P. D. Robinson \\ McMaster University Evidence-based Practice Center, Hamilton, \\ ON, Canada
}

alone for patients with single metastatic brain tumors who have a KPS $\geq 70$.

Level 1 Single-dose SRS along with WBRT is superior in terms of local tumor control and maintaining functional status when compared to WBRT alone for patients with 1-4 metastatic brain tumors who have a KPS $\geq 70$.

Level 2 Single-dose SRS along with WBRT may lead to significantly longer patient survival than WBRT alone for patients with 2-3 metastatic brain tumors.

Level 3 There is class III evidence demonstrating that single-dose SRS along with WBRT is superior to WBRT alone for improving patient survival for patients with single or multiple brain metastases and a KPS $<70$.

M. Ammirati

Department of Neurosurgery, Ohio State University Medical Center, Columbus, OH, USA

\section{S. Cobbs}

Department of Neurosciences, California Pacific Medical Center, San Francisco, CA, USA

\section{E. Gaspar}

Department of Radiation Oncology, University of ColoradoDenver, Denver, CO, USA

\section{J. S. Loeffler}

Department of Radiation Oncology, Massachusetts General Hospital, Boston, MA, USA

\section{McDermott}

Department of Neurosurgery, University of California

San Francisco, San Francisco, CA, USA

\section{P. Mehta}

Department of Human Oncology, University of Wisconsin School of Public Health and Medicine, Madison, WI, USA 
T. Mikkelsen $\cdot$ S. N. Kalkanis $(\square)$

Department of Neurology, Henry Ford Health System, 2799

West Grand Blvd, K-11, Detroit, MI 48202, USA

e-mail: kalkanis@neuro.hfh.edu

\section{J. J. Olson}

Department of Neurosurgery, Emory University School of Medicine, Atlanta, GA, USA

N. A. Paleologos

Department of Neurology, Northshore University Health

System, Evanston, IL, USA

R. A. Patchell

Department of Neurology, Barrow Neurological Institute,

Phoenix, AZ, USA

T. C. Ryken

Department of Neurosurgery, Iowa Spine and Brain Institute, Iowa City, IA, USA 